\title{
On the fixed volume discrepancy of the Fibonacci sets in the integral norms
}

\author{
V.N. Temlyakov* and M. Ullrich ${ }^{\dagger}$
}

\begin{abstract}
This paper is devoted to the study of a discrepancy-type characteristic - the fixed volume discrepancy - of the Fibonacci point set in the unit square. It was observed recently that this new characteristic allows us to obtain optimal rate of dispersion from numerical integration results. This observation motivates us to thoroughly study this new version of discrepancy, which seems to be interesting by itself. The new ingredient of this paper is the use of the average over the shifts of hat functions instead of taking the supremum over the shifts. We show that this change in the setting results in an improvement of the upper bound for the smooth fixed volume discrepancy, similarly to the well-known results for the usual $L_{p}$-discrepancy. Interestingly, this shows that "bad boxes" for the usual discrepancy cannot be "too small". The known results on smooth discrepancy show that the obtained bounds cannot be improved in a certain sense.
\end{abstract}

\section{Introduction}

This paper is devoted to the study of a discrepancy-type characteristic - the fixed volume discrepancy - of a point set in the unit square $\Omega_{2}:=[0,1)^{2}$. We refer the reader to the following books and survey papers on discrepancy theory and numerical integration [2], [7], 8], [19], 3] [5], [15], and [20]. Recently, an important new observation was made in [16]. It claims that a new

${ }^{*}$ University of South Carolina, USA; Steklov Institute of Mathematics and Lomonosov Moscow State University, Russia.

${ }^{\dagger}$ Johannes Kepler University Linz, Austria. 
version of discrepancy - the $r$-smooth fixed volume discrepancy - allows us to obtain optimal rate of dispersion from numerical integration results (see [1, 4, 6, 9, 11, 13, 21, 22, 23, for some recent results on dispersion). This observation motivates us to thoroughly study this new version of discrepancy, which seems to be interesting by itself.

The $r$-smooth fixed volume discrepancy takes into account two characteristics of a smooth hat function $h_{B}^{r}$ - its smoothness $r$ and the volume of its support $v:=\operatorname{vol}(B)$ (see the definition of $h_{B}^{r}$ below). The new ingredient of this paper is the use of the $L_{p}, 1 \leq p<\infty$, average over the shifts of hat functions instead of taking the supremum over the shifts. We show that this change in the setting of the problem results in an improvement of the upper bound for the $r$-smooth fixed volume discrepancy of the special sets of points - the Fibonacci point sets. For these sets with $b_{n}$ elements (see below), we get $\left(\log \left(b_{n} v\right)\right)^{1 / 2}$ for $1 \leq p<\infty$, instead of $\log \left(b_{n} v\right)$ for $p=\infty$. The known results on $r$-smooth discrepancy show that both bounds cannot be improved in a certain sense (see the end of Introduction for a detailed discussion). The new results are only for the Fibonacci point sets, i.e., in dimension 2, and for $L_{p}$-averaging in the periodic setting, i.e., with respect to the torus geometry. However, we present the corresponding definitions and some known results in a general setting on the unit cube $\Omega_{d}:=[0,1)^{d}$. We now proceed to a formal description of the problem setting and to formulation of the results.

Denote by $\chi_{[a, b)}(x)$ a univariate characteristic function $($ on $\mathbb{R}$ ) of the interval $[a, b)$ and, for $r=1,2,3, \ldots$, we inductively define

$$
h_{u}^{1}(x):=\chi_{[-u / 2, u / 2)}(x)
$$

and

$$
h_{u}^{r}(x):=h_{u}^{r-1}(x) * h_{u}^{1}(x)
$$

where

$$
f(x) * g(x):=\int_{\mathbb{R}} f(x-y) g(y) d y .
$$

Note that $h_{u}^{2}$ is the hat function, i.e., $h_{u}^{2}(x)=\max \{u-|x|, 0\}$.

Let $\Delta_{t} f(x):=f(x)-f(x+t)$ be the first difference. We say that a univariate function $f$ has smoothness 1 in $L_{1}$ if $\left\|\Delta_{t} f\right\|_{1} \leq C|t|$ for some absolute constant $C<\infty$. In case $\left\|\Delta_{t}^{r} f\right\|_{1} \leq C|t|^{r}$, where $\Delta_{t}^{r}:=\left(\Delta_{t}\right)^{r}$ is the $r$ th difference operator, $r \in \mathbb{N}$, we say that $f$ has smoothness $r$ in $L_{1}$. Then, $h_{u}^{r}(x)$ has smoothness $r$ in $L_{1}$ and has support $(-r u / 2, r u / 2)$. 
For a box $B$ of the form

$$
B=\prod_{j=1}^{d}\left[z_{j}-r u_{j} / 2, z_{j}+r u_{j} / 2\right)
$$

define

$$
h_{B}^{r}(\mathbf{x}):=h_{\mathbf{u}}^{r}(\mathbf{x}-\mathbf{z}):=\prod_{j=1}^{d} h_{u_{j}}^{r}\left(x_{j}-z_{j}\right) .
$$

We begin with the non-periodic $r$-smooth fixed volume discrepancy introduced and studied in [16].

Definition 1.1. Let $r \in \mathbb{N}, v \in(0,1]$ and $\xi:=\left\{\xi^{\mu}\right\}_{\mu=1}^{m} \subset[0,1)^{d}$ be a point set. We define the $r$-smooth fixed volume discrepancy with equal weights as

$$
D^{r}(\xi, v):=\sup _{B \subset \Omega_{d}: \operatorname{vol}(B)=v}\left|\int_{\Omega_{d}} h_{B}^{r}(\mathbf{x}) d \mathbf{x}-\frac{1}{m} \sum_{\mu=1}^{m} h_{B}^{r}\left(\xi^{\mu}\right)\right| .
$$

The optimized version of the r-smooth fixed volume discrepancy is defined as follows

$$
D^{r, o}(\xi, v):=\inf _{\lambda_{1}, \ldots, \lambda_{m}} \sup _{B \subset \Omega_{d}: v o l(B)=v}\left|\int_{\Omega_{d}} h_{B}^{r}(\mathbf{x}) d \mathbf{x}-\sum_{\mu=1}^{m} \lambda_{\mu} h_{B}^{r}\left(\xi^{\mu}\right)\right| .
$$

Clearly, we have $D^{r, o}(\xi, v) \leq D^{r}(\xi, v)$.

It is well known that the Fibonacci cubature formulas are optimal in the sense of order for numerical integration of different kind of smoothness classes of functions of two variables, see e.g. [5, 14, 19]. We present a result from [16], which shows that the Fibonacci point set has good fixed volume discrepancy.

Let $\left\{b_{n}\right\}_{n=0}^{\infty}, b_{0}=b_{1}=1, b_{n}=b_{n-1}+b_{n-2}, n \geq 2$, be the Fibonacci numbers. Denote the $n$th Fibonacci point set by

$$
\mathcal{F}_{n}:=\left\{\left(\mu / b_{n},\left\{\mu b_{n-1} / b_{n}\right\}\right): \mu=1, \ldots, b_{n}\right\} .
$$

In this definition $\{a\}$ is the fractional part of the number $a$. The cardinality of the set $\mathcal{F}_{n}$ is equal to $b_{n}$. In [16] we proved the following upper bound. 
Theorem 1.1. Let $r \geq 2$. There exist constants $c, C>0$ such that for any $v \geq c / b_{n}$ we have

$$
D^{r}\left(\mathcal{F}_{n}, v\right) \leq C \frac{\log \left(b_{n} v\right)}{b_{n}^{r}} .
$$

The main object of our interest in this paper is the periodic $r$-smooth $L_{p^{-}}$ discrepancy of the Fibonacci point sets. For this, we define the periodization $\tilde{f}$ (with period 1 in each variable) of a function $f \in L_{1}\left(\mathbb{R}^{d}\right)$ with a compact support by

$$
\tilde{f}(\mathbf{x}):=\sum_{\mathbf{m} \in \mathbb{Z}^{d}} f(\mathbf{m}+\mathbf{x})
$$

and, for each $B \subset[0,1)^{d}$, we let $\tilde{h}_{B}^{r}$ be the periodization of $h_{B}^{r}$ from (1.2).

We now define the periodic $r$-smooth $L_{p}$-discrepancy.

Definition 1.2. For $r \in \mathbb{N}, 1 \leq p \leq \infty$ and $v \in(0,1]$ define the periodic $r$-smooth fixed volume $L_{p}$-discrepancy of a point set $\xi$ by

$$
\tilde{D}_{p}^{r}(\xi, v):=\sup _{B \subset \Omega_{d}: v o l(B)=v}\left\|\int_{\Omega_{d}} \tilde{h}_{B}^{r}(\mathbf{x}-\mathbf{z}) d \mathbf{x}-\frac{1}{m} \sum_{\mu=1}^{m} \tilde{h}_{B}^{r}\left(\xi^{\mu}-\mathbf{z}\right)\right\|_{p}
$$

where the $L_{p}$-norm is taken with respect to $\mathbf{z}$ over the unit cube $\Omega_{d}=[0,1)^{d}$. Analogously to (1.4) we may define the optimized version $\tilde{D}_{p}^{r, o}(\xi, v)$.

In the case of $p=\infty$ this concept was introduced and studied in [17].

We prove the following upper bound for $1 \leq p<\infty$.

Theorem 1.2. Let $r \in \mathbb{N}$ and $1 \leq p<\infty$. There exist constants $c, C>0$ such that for any $v \geq c / b_{n}$ we have

$$
\tilde{D}_{p}^{r}\left(\mathcal{F}_{n}, v\right) \leq C \frac{\sqrt{\log \left(b_{n} v\right)}}{b_{n}^{r}} .
$$

In the case $p=\infty$ we prove a weaker upper bound.

Theorem 1.3. Let $r \geq 2$. There exist constants $c, C>0$ such that for any $v \geq c / b_{n}$ we have

$$
\tilde{D}_{\infty}^{r}\left(\mathcal{F}_{n}, v\right) \leq C \frac{\log \left(b_{n} v\right)}{b_{n}^{r}} .
$$


We now give some comments, which show that Theorems 1.2 and 1.3 cannot be improved in a certain sense. We do not know if Theorems 1.2 and 1.3 are sharp for all $v \geq c / b_{n}$. The known results show that these theorems are sharp in some cases for the supremum over $v$. The following quantities

$$
\tilde{D}_{p, \infty}^{r, o}(\xi):=\sup _{v} \tilde{D}_{p}^{r, o}(\xi, v)
$$

have been studied in [17 and [18. We cite some results from there. The following lower bound follows from stronger results in [18]. Let $r, d \in \mathbb{N}$. Then for any point set $\xi \subset \Omega_{d}$ with $\# \xi=m$ we have

$$
\tilde{D}_{2, \infty}^{r, o}(\xi) \geq C(r, d) m^{-r}(\log m)^{(d-1) / 2}, \quad C(r, d)>0 .
$$

Under an extra assumption on $r$, namely, assuming that $r$ is an even number, we can derive an extended to $p>1$ inequality (1.7) for $\tilde{D}_{p, \infty}^{r, o}(\xi)$ from [18]. In the case $r=1$ the quantity under consideration corresponds to the classical (non-smooth) discrepancy, and the above mentioned bounds were already proven in [10, 12].

For $p=\infty$ the following result was proved in [17]. For any point set $\xi \subset \Omega_{d}$ with $\# \xi=m$ we have for even integers $r$ that

$$
\tilde{D}_{\infty}^{r}(\xi) \geq C(r, d) m^{-r}(\log m)^{d-1}
$$

with a positive constant $C(r, d)$. This result even holds if we allow weights (as in the optimized version) $\lambda_{1}, \ldots, \lambda_{m}$ satisfying condition

$$
\sum_{\mu=1}^{m}\left|\lambda_{\mu}\right| \leq B
$$

for some fixed $B<\infty$.

Finally, let us add that Theorems 1.2 and 1.3 show that the "bad boxes", i.e., the boxes that fulfill the lower bounds (1.7) or (1.8), must have volume at least $m^{-1+\delta}$ for some fixed $\delta>0$. This is interesting as one might think that boxes of volume at most $(\log m)^{c} / m$ (for some large $c$ ) may already suffice.

\section{Proofs of Theorems 1.2 and 1.3}

The proofs of both theorems go along the same lines. We give a detailed proof of Theorem 1.2 and point out a change of this proof, which gives Theorem 
1.3. For continuous functions of two variables, which are 1-periodic in each variable, define cubature formulas

$$
\Phi_{n}(f):=b_{n}^{-1} \sum_{\mu=1}^{b_{n}} f\left(\mu / b_{n},\left\{\mu b_{n-1} / b_{n}\right\}\right),
$$

called the Fibonacci cubature formulas. Denote

$$
\mathbf{y}^{\mu}:=\left(\mu / b_{n},\left\{\mu b_{n-1} / b_{n}\right\}\right), \quad \mu=1, \ldots, b_{n},
$$

and

$$
\Phi(\mathbf{k}):=\Phi_{n}\left(e^{i 2 \pi(\mathbf{k}, \mathbf{x})}\right)=b_{n}^{-1} \sum_{\mu=1}^{b_{n}} e^{i 2 \pi\left(\mathbf{k}, \mathbf{y}^{\mu}\right)}
$$

Note that

$$
\Phi_{n}(f)=\sum_{\mathbf{k}} \hat{f}(\mathbf{k}) \Phi(\mathbf{k}), \quad \hat{f}(\mathbf{k}):=\int_{[0,1)^{2}} f(\mathbf{x}) e^{-i 2 \pi(\mathbf{k}, \mathbf{x})} d \mathbf{x}
$$

where for the sake of simplicity we may assume that $f$ is a trigonometric polynomial. It is clear that (2.1) holds for $f$ with absolutely convergent Fourier series.

It is easy to see that the following relation holds

$$
\Phi(\mathbf{k})=\left\{\begin{array}{lll}
1 & \text { for } & \mathbf{k} \in L(n) \\
0 & \text { for } & \mathbf{k} \notin L(n)
\end{array}\right.
$$

where

$$
L(n):=\left\{\mathbf{k}=\left(k_{1}, k_{2}\right) \in \mathbb{Z}^{2}: k_{1}+b_{n-1} k_{2} \equiv 0 \quad\left(\bmod b_{n}\right)\right\} .
$$

For $N \in \mathbb{N}$ define the hyperbolic cross (in dimension 2) by

$$
\Gamma(N):=\left\{\mathbf{k}=\left(k_{1}, k_{2}\right) \in \mathbb{Z}^{2}: \prod_{j=1}^{2} \max \left(\left|k_{j}\right|, 1\right) \leq N\right\} .
$$

The following lemma is well known (see, for instance, [19], p.274).

Lemma 2.1. There exists an absolute constant $\gamma>0$ such that for any $n>2$ we have

$$
\Gamma\left(\gamma b_{n}\right) \cap(L(n) \backslash\{\mathbf{0}\})=\varnothing .
$$


Considering our (univariate) test functions $h_{u}^{r}$ we obtain by the properties of convolution that

$$
\hat{h}_{u}^{r}(y)=\hat{h}_{u}^{r-1}(y) \hat{h}_{u}^{1}(y), \quad y \in \mathbb{R}
$$

which implies for $y \neq 0$

$$
\hat{h}_{u}^{r}(y)=\left(\frac{\sin (\pi y u)}{\pi y}\right)^{r}
$$

Therefore,

$$
\left|\hat{\tilde{h}}_{u}^{r}(k)\right| \leq \min \left(|u|^{r}, \frac{1}{|k|^{r}}\right)=\left(\frac{|u|}{k^{\prime}}\right)^{r / 2} \min \left(\left|k^{\prime} u\right|^{r / 2}, \frac{1}{|k u|^{r / 2}}\right),
$$

where $k^{\prime}:=\max \{1,|k|\}$. (Here, we used for a moment $\hat{h}$ for the Fourier transform of $h$ on $\mathbb{R}$. This should not lead to any confusion.)

We now proceed with some considerations in arbitrary dimension $d$. It is convenient for us to use the following abbreviated notation for the product

$$
\operatorname{pr}(\mathbf{u}):=\operatorname{pr}(\mathbf{u}, d):=\prod_{j=1}^{d} u_{j}
$$

For $B \subset \Omega_{d}$ of the form (1.1) and $\mathbf{z} \in \Omega_{d}$, we have

$$
\hat{\tilde{h}}_{B+\mathbf{z}}^{r}(\mathbf{k})=e^{-i 2 \pi(\mathbf{k}, \mathbf{z})} \hat{\tilde{h}}_{B}^{r}(\mathbf{k}),
$$

where $\tilde{h}_{B+\mathbf{z}}^{r}(\mathbf{x}):=\tilde{h}_{B}^{r}(\mathbf{x}-\mathbf{z})$, see (1.2). Therefore, we obtain from the above that

$$
\left|\hat{\tilde{h}}_{B}^{r}(\mathbf{k})\right| \leq \prod_{j=1}^{d}\left(\frac{\left|u_{j}\right|}{k_{j}^{\prime}}\right)^{r / 2} \min \left(\left|k_{j}^{\prime} u_{j}\right|^{r / 2}, \frac{1}{\left|k_{j} u_{j}\right|^{r / 2}}\right) .
$$

For $\mathbf{s} \in \mathbb{N}_{0}^{d}$, we define

$$
\rho(\mathbf{s}):=\left\{\mathbf{k} \in \mathbb{Z}^{d}:\left[2^{s_{j}-1}\right] \leq\left|k_{j}\right|<2^{s_{j}}, \quad j=1, \ldots, d\right\},
$$

where $[a]$ denotes the integer part of $a$, and obtain, for $\mathbf{k} \in \rho(\mathbf{s})$, that

$$
\left|\hat{\tilde{h}}_{B}^{r}(\mathbf{k})\right| \leq H_{B}^{r}(\mathbf{s}):=\left(\frac{p r(\mathbf{u})}{2^{\|\mathbf{s}\|_{1}}}\right)^{r / 2} \prod_{j=1}^{d} \min \left(\left(2^{s_{j}} u_{j}\right)^{r / 2}, \frac{1}{\left(2^{s_{j}} u_{j}\right)^{r / 2}}\right) .
$$


Later we will need certain sums of these quantities. First, consider

$$
\sigma_{\mathbf{u}}^{r}(t):=\sum_{\|\mathbf{s}\|_{1}=t} \prod_{j=1}^{d} \min \left(\left(2^{s_{j}} u_{j}\right)^{r / 2}, \frac{1}{\left(2^{s_{j}} u_{j}\right)^{r / 2}}\right), \quad t \in \mathbb{N}_{0} .
$$

The following technical lemma is part (I) from [16, Lemma 6.1].

Lemma 2.2. Let $r>0, t \in \mathbb{N}$ and $\mathbf{u} \in(0,1 / 2]^{d}$ be such that $\operatorname{pr}(\mathbf{u}) \geq 2^{-t}$. Then, we have

$$
\sigma_{\mathbf{u}}^{r}(t) \leq C(d) \frac{\left(\log \left(2^{t+1} \operatorname{pr}(\mathbf{u})\right)\right)^{d-1}}{\left(2^{t} \operatorname{pr}(\mathbf{u})\right)^{r / 2}}
$$

This lemma and (2.4) imply that

$$
\sum_{\|\mathbf{s}\|_{1}=t} H_{B}^{r}(\mathbf{s})^{2} \leq C_{1} 2^{-2 r t}\left(\log \left(2^{t} v\right)\right)^{d-1}
$$

where $v:=\operatorname{vol}(B)=r^{d} \operatorname{pr}(\mathbf{u})$, for all $r \geq 1$ and all $t \in \mathbb{N}_{0}$ with $v \geq r^{d} 2^{-t+1}$ and an absolute constant $C_{1}<\infty$.

Additionally, we need a result from harmonic analysis - a corollary of the Littlewood-Paley theorem. Denote

$$
\delta_{\mathbf{s}}(f, \mathbf{x}):=\sum_{\mathbf{k} \in \rho(\mathbf{s})} \hat{f}(\mathbf{k}) e^{i 2 \pi(\mathbf{k}, \mathbf{x})} .
$$

Then it is known that for $p \in[2, \infty)$ one has

$$
\|f\|_{p} \leq C(d, p)\left(\sum_{\mathbf{s} \in \mathbb{N}_{0}^{d}}\left\|\delta_{\mathbf{s}}(f)\right\|_{p}^{2}\right)^{1 / 2}
$$

Note that in the proof of Theorem 1.3 we use the simple triangle inequality

$$
\|f\|_{\infty} \leq \sum_{\mathbf{s}}\left\|\delta_{\mathbf{s}}(f)\right\|_{\infty}
$$

instead of (2.6). 
We are now considering the case $d=2$. Let us define

$$
E_{B}^{r}(\mathbf{z}):=\frac{1}{b_{n}} \sum_{\mu=1}^{b_{n}} \tilde{h}_{B}^{r}\left(\mathbf{y}^{\mu}-\mathbf{z}\right)-\int_{[0,1)^{d}} \tilde{h}_{B}^{r}(\mathbf{x}) d \mathbf{x}
$$

such that

$$
\tilde{D}_{p}^{r}\left(\mathcal{F}_{n}, v\right)=\sup _{B \subset \Omega_{d}: \operatorname{vol}(B)=v}\left\|E_{B}^{r}\right\|_{p}
$$

By formulas (2.1), (2.2) and (2.3) we obtain

$$
E_{B}^{r}(\mathbf{z})=\sum_{\mathbf{k} \neq 0} \hat{\tilde{h}}_{B}^{r}(\mathbf{k}) \Phi(\mathbf{k}) e^{-i 2 \pi(\mathbf{k}, \mathbf{z})}=\sum_{\mathbf{k} \in L(n) \backslash\{0\}} \hat{\tilde{h}}_{B}^{r}(\mathbf{k}) e^{-i 2 \pi(\mathbf{k}, \mathbf{z})} .
$$

It is apparent from (2.6) that it remains to bound $\left\|\delta_{s}\left(E_{B}^{r}\right)\right\|_{p}$.

If $t \neq 0$ is such that $2^{t} \leq \gamma b_{n}$ then for $\mathbf{s}$ with $\|\mathbf{s}\|_{1}=t$ we have $\rho(\mathbf{s}) \subset$ $\Gamma\left(\gamma b_{n}\right)$. Lemma 2.1 then implies that $\Phi(\mathbf{k})=0$ for $\mathbf{k} \in \rho(\mathbf{s})$ and, therefore, $\delta_{s}\left(E_{B}^{r}\right)=0$. Let $t_{0} \in \mathbb{N}$ be the smallest number satisfying $2^{t_{0}}>\gamma b_{n}$, i.e., $t_{0} \geq \log \left(b_{n}\right)-c$ for some $c<\infty$. Then, from (2.6) for $p \in[2, \infty)$, we have

$$
\left\|E_{B}^{r}\right\|_{p} \leq C(p)\left(\sum_{t=t_{0}}^{\infty} \sum_{\|\mathbf{s}\|_{1}=t}\left\|\delta_{s}\left(E_{B}^{r}\right)\right\|_{p}^{2}\right)^{1 / 2} .
$$

Moreover, Lemma 2.1 implies that for $t \geq t_{0}$ we have

$$
\#(\rho(\mathbf{s}) \cap L(n)) \leq C_{2} 2^{t-t_{0}}, \quad\|\mathbf{s}\|_{1}=t .
$$

By Parselval's identity we obtain

$$
\left\|\delta_{s}\left(E_{B}^{r}\right)\right\|_{2}=\sqrt{\sum_{\mathbf{k} \in \rho(\mathbf{s}) \cap L(n)}\left|\hat{\tilde{h}}_{B}^{r}(\mathbf{k})\right|^{2}} \leq \sqrt{\#(\rho(\mathbf{s}) \cap L(n))} \cdot H_{B}^{r}(\mathbf{s})
$$

and, by the triangle inequality,

$$
\left\|\delta_{s}\left(E_{B}\right)\right\|_{\infty} \leq \#(\rho(\mathbf{s}) \cap L(n)) \cdot H_{B}^{r}(\mathbf{s})
$$

Hence, using the inequality

$$
\|f\|_{p} \leq\|f\|_{2}^{2 / p}\|f\|_{\infty}^{1-2 / p}
$$


for $2 \leq p \leq \infty$, we get

$$
\left\|\delta_{s}\left(E_{B}^{r}\right)\right\|_{p} \leq(\#(\rho(\mathbf{s}) \cap L(n)))^{1-1 / p} \cdot H_{B}^{r}(\mathbf{s}) .
$$

Combining this with (2.5) for $d=2,(2.9)$ and (2.10), we finally obtain for all $v=\operatorname{vol}(B) \geq 2 r^{d} 2^{-t_{0}}$ and $p \in[2, \infty)$ that

$$
\begin{aligned}
\left\|E_{B}^{r}\right\|_{p} & \leq C\left(\sum_{t=t_{0}}^{\infty} 2^{2\left(t-t_{0}\right)(1-1 / p)} \sum_{\|\mathbf{s}\|_{1}=t} H_{B}^{r}(\mathbf{s})^{2}\right)^{1 / 2} \\
& \leq C^{\prime}\left(\sum_{t=t_{0}}^{\infty} 2^{2\left(t-t_{0}\right)(1-1 / p)} 2^{-2 r t} \log \left(2^{t} v\right)\right)^{1 / 2} \\
& =C^{\prime} 2^{-r t_{0}}\left(\sum_{t=0}^{\infty} 2^{2 t(1-1 / p-r)} \log \left(2^{t+t_{0}} v\right)\right)^{1 / 2} \\
& \leq C^{\prime \prime} 2^{-r t_{0}} \sqrt{\log \left(2^{t_{0}} v\right)}\left(\sum_{t=0}^{\infty} t 2^{2 t(1-1 / p-r)}\right)^{1 / 2} .
\end{aligned}
$$

Using $t_{0} \geq \log \left(b_{n}\right)-c$ and that $\left\|E_{B}^{r}\right\|_{p} \leq\left\|E_{B}^{r}\right\|_{2}$ for $p<2$, this implies Theorem 1.2. (Here, we used that clearly $r>1-1 / p$ for $p<\infty$.)

As we pointed out above, in the proof of Theorem 1.3 we use inequality (2.7) instead of (2.6). Moreover we use

$$
\sum_{\|\mathbf{s}\|_{1}=t} H_{B}^{r}(\mathbf{s}) \leq C_{1} 2^{-r t} \log \left(2^{t} v\right),
$$

for all $r \geq 1$ instead of (2.5)). However, note that we need $r>1$ for the last series in the above computation to be finite. This implies

$$
\left\|E_{B}^{r}\right\|_{\infty} \leq C b_{n}^{-r} \log \left(b_{n} v\right) .
$$

Acknowledgment. The work was supported by the Russian Federation Government Grant No. 14.W03.31.0031.

\section{References}

[1] C. Aistleitner, A. Hinrichs, and D. Rudolf, On the size of the largest empty box amidst a point set, Discrete Appl. Math. 230 (2017), 146150. 
[2] J. Beck and W. Chen, Irregularities of distribution, Cambridge University Press, Cambridge, 1987.

[3] D. Bilyk, Roth's Orthogonal Function Method in Discrepancy Theory and Some New Connections, in Panorama of Discrepancy Theory, Lecture Notes in Mathematics 2107, Springer-Verlag, London, 2014, $71-158$.

[4] S. Breneis and A. Hinrichs, Fibonacci lattices have minimal dispersion on the two-dimensional torus, preprint, arXiv:1905.03856.

[5] Ding Dũng, V.N. Temlyakov, and T. Ullrich, Hyperbolic Cross Approximation, arXiv:1601.03978v2 [math.NA] 2 Dec 2016.

[6] A. Dumitrescu and M. Jiang, On the largest empty axis-parallel box amidst $n$ points, Algorithmica, 66 (2013), 225-248.

[7] J. Matousek, Geometric Discrepancy, Springer, 1999.

[8] E. Novak and H. Woźniakowski. Tractability of multivariate problems. Volume II: Standard information for functionals, volume 12 of EMS Tracts in Mathematics. European Mathematical Society (EMS), Zürich, 2010.

[9] G. Rote and F. Tichy, Quasi-Monte Carlo methods and the dispersion of point sequences, Math. Comput. Modelling, 23 (1996), 9-23.

[10] K. F. Roth, On irregularities of distribution, Mathematika 1 (1954), 73-79.

[11] D. Rudolf, An upper bound of the minimal dispersion via delta covers, Contemporary Computational Mathematics - a Celebration of the 80th Birthday of Ian Sloan. Springer-Verlag, 2018.

[12] W. M. Schmidt, Irregularities of distribution. X. In: Number theory and algebra, pp. 311-329. Academic Press, New York, 1977.

[13] J. Sosnovec, A note on minimal dispersion of point sets in the unit cube, European J. Combin. 69 (2018), 255259.

[14] V.N. Temlyakov, Approximation of periodic functions, Nova Science Publishers, Inc., New York., 1993. 
[15] V.N. Temlyakov, Cubature formulas and related questions, J. Complexity 19 (2003), 352-391.

[16] V.N. Temlyakov, Smooth fixed volume discrepancy, dispersion, and related problems, J. Approx. Theory, 237 (2019), 113-134; arXiv:1709.08158v2 [math.NA] 4 Oct 2017.

[17] V.N. Temlyakov, Fixed volume discrepancy in the periodic case, arXiv:1710.11499v1 [math.NA] 30 Oct 2017.

[18] V.N. Temlyakov, Remarks on numerical integration, discrepancy, and diaphony, arXiv:1711.07017v1 [math.NA] 19 Nov 2017.

[19] V.N. Temlyakov, Multivariate approximation, Cambridge University Press, 2018.

[20] V.N. Temlyakov, Connections between numerical integration, discrepancy, dispersion, and universal discretization, arXiv:1812.04489v1 [math.NA] 9 Dec 2018.

[21] M. Ullrich, A lower bound for the dispersion on the torus, Math. Comput. Simulation 143 (2018), 186-190.

[22] M. Ullrich, A note on the dispersion of admissible lattices, Discrete Appl. Math. 257 (2019), 385387.

[23] M. Ullrich and J. Vybíral, An upper bound on the minimal dispersion, J. Complexity 45 (2018), 120-126. 